\title{
Dermatosparaxia em um ovino: achados clínicos, anatomopatológicos e moleculares
}

\author{
Dermatosparaxis in a Sheep: Clinical, Anatomopathological, and Molecular Findings \\ Mariana Correia Oliveira', Bruna Patrícia Siqueira Raimundo², Carla Ribeiro Silveira', \\ Bruno Gonçalves de Souza ${ }^{2}$, Daniel Guimarães Ubiali', Danilo Giorgi Abranches de Andrade, \\ José Paes de Oliveira-Filho ${ }^{3} \&$ Marilene de Farias Brito ${ }^{1}$
}

\begin{abstract}
Background: Dermatosparaxis is an autosomal recessive genetic disease that affects the connective tissue of animals. Collagen proteins form fibrillar structures that provide strength and structure to the extracellular matrix of tissues and organs in the body. Therefore, changes in collagen synthesis result in hyperextensibility and skin fragility. Similar to dermatosparaxis in animals, some cases of Ehlers-Danlos syndrome type VIIC have been reported in humans. The objective of this study was to describe the clinical, anatomopathological, and molecular findings of a case of dermatosparaxis in a sheep bred by crossing the Santa Inês and White Dorper breeds.

Case: A case of dermatosparaxis was reported in a 20-day-old female sheep, a cross between the Santa Inês and White Dorper breeds. The sheep exhibited hyperextensibility of the skin with minimal tension and increased joint volume shortly after birth. Because of clinical worsening and the poor prognosis of the disease, the owners opted for euthanasia. The necropsy revealed large ulcerated areas in several parts of the body, including the cervical, sternal, scapular, and thoracic regions, and the inner and outer thighs. The skin was extremely easy to be removed during the necropsy and the use of a scalpel was not necessary. Extensive areas of hemorrhage were observed in the subcutaneous tissue and small intestine. The histopathological findings of the skin lesions evidenced the presence of dermatosparaxis, particularly regarding the disorganization of collagen fibers. The tests involving Masson's trichrome staining, picrosirius red staining, and Gomori's trichrome staining under polarized light evidence of collagen fiber dysplasia.

Discussion: The diagnosis of dermatosparaxis in this study was based on clinical, anatomopathological, and molecular analysis. Molecular diagnosis was confirmed by identification of SNP c.421G $>$ T on the ADAMTS2 gene in both the mother and the sheep under study. In humans, in addition to hyperextensibility and skin fragility, other rare clinical signs include ligamentous laxity, bone abnormalities, vascular abnormalities, uterine rupture, ocular abnormalities, and extensive areas of hemorrhage in the intestine. In this case, the humeral-radial-ulnar joints of both thoracic limbs were substantially enlarged and, when sectioned, a moderate amount of dark yellow fluid was released. Dermatosparaxis is well documented in the White Dorper sheep breed. In the present study, the genetic test revealed that the mother (Santa Inês breed) was heterozygous and phenotypically normal. All the rams in the animal herd (White Dorper breed) were phenotypically normal. Extensive hemorrhage in organs of the abdominal cavity and the high likelihood of skin rupture have been reported in other species that are affected by the disease. The histopathological findings of cutaneous lesions in this sheep agree with the descriptions in the literature on the disorganization of collagen fibers. The tests involving Masson's trichrome staining, Gomori's trichrome, and picrosirius red staining under polarized light were crucial for providing evidence of collagen fiber dysplasia. Even when phenotypically normal, the heterozygous sheep in the herd can produce recessive homozygous animals, which may be clinically affected, mainly, if there is a history of uncontrolled crossing in the herd.
\end{abstract}

Keywords: collagen, skin fragility, dermatopathy, mutation, sheep.

Descritores: colágeno, fragilidade cutânea, dermatopatia, mutação, ovino. 


\section{INTRODUÇÃO}

Dermatosparaxia é uma doença genética de caráter autossômico recessivo que afeta o tecido conjuntivo, caracterizada clinicamente por hiperextensibilidade e fragilidade cutânea observada por lacerações ao mínimo trauma $[18,20]$.

Em humanos, a doença análoga à dermatosparaxia em animais, é chamada síndrome de Ehlers-Danlos tipo VIIC, e ambas levam à formação de fibrilas de colágeno anormais que não fornecem a resistência necessária à pele $[11,15,20]$.

A dermatosparaxia tem sido relatada em ovinos [12,17-19], bovinos [9], búfalos [10], equinos [4,8,21], cães $[5,14]$ e gatos $[1,7,16]$.

Sinais clínicos e estudos histopatológicos da estrutura do colágeno são importantes para identificar esta doença [16], porém os estudos moleculares permitem confirmar o diagnóstico da doença [2].

Os objetivos deste estudo foram descrever os achados clínicos, anatomopatológicos e moleculares de um caso de dermatosparaxia em ovino cruzamento das raças Santa Inês e White Dorper.

\section{CASO}

Uma ovelha, de 20 dias de idade, cruzamento das raças Santa Inês e White Dorper, foi encaminhada do município do Rio de Janeiro para o Setor de Grandes Animais do Hospital Veterinário da Universidade Federal Rural do Rio de Janeiro (HVGA-UFRRJ) com diversas lesões lacerantes por todo o corpo e histórico de aparecimento cinco dias após o nascimento.

Ao exame clínico percebeu-se que as lesões cutâneas pareciam ter evoluções distintas, onde se via apenas ulcerações, ou ferimentos inflamados, com bordas necróticas, ou com grandes áreas de perda cutânea e até muscular. Foram realizadas tricotomias peri-lesionais, limpeza local com solução fisiológica, seguida de aplicação de pomada contendo antibióticos e cicatrizantes. Notou-se que a pele do ovino como um todo possuía hiperextensibilidade, além de romper-se com a simples tração. As articulações apendiculares estavam quentes e aumentadas em volume (Figura 1).

Ao exame físico, a auscultação respiratória revelou frequência de 32 incursões por minuto (ipm), frequência cardíaca de 180 batimentos por minuto (bpm), temperatura retal de $38,4^{\circ} \mathrm{C}$, mucosas aparentes pálidas, tempo de preenchimento capilar aumentado e turgor de pele diminuído.

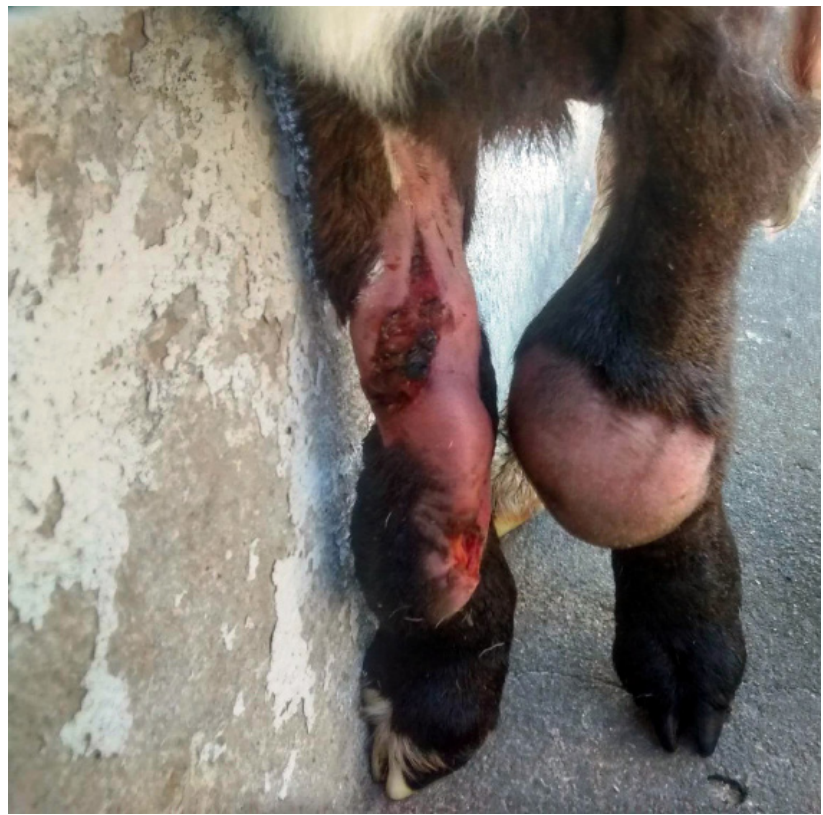

Figura 1. Membro torácico direito com extensas áreas ulceradas. Membro torácico esquerdo com acentuado aumento de volume da articulação rádiocarpo-metacarpiana.

Foram realizados exames hematológicos que não demonstraram alterações. A fim de se evitar infecção secundária, foi instituído tratamento sistêmico com antibiótico à base de penicilina potássica ${ }^{1}$ (dose: 30.000 U.I./ $\mathrm{Kg})$, suplementação parenteral de glicose ${ }^{2}(0,5 \mathrm{~g} / \mathrm{Kg})$ e sais minerais e complexos vitamínicos (Bioxan $\left.{ }^{\circledR}\right)^{1}$, além de Maxicam ${ }^{\circledR}{ }^{1}(0,5 \mathrm{mg} / \mathrm{Kg}$ por via intravenosa; SID) e soro antitetânico ${ }^{3}$ (5.000 U.I.). O tratamento tópico foi realizado com a lavagem com solução degermante de clorexidina $2 \%$ e pomada à base de sulfadiazina de prata $^{2}$. Após cinco dias de internação e tratamento, as lesões de pele não apresentaram melhora e novas lesões surgiram durante o manejo das feridas. Suspeitando-se da possibilidade de tratar-se de dermatosparaxia, o proprietário foi questionado sobre aspectos zootécnicos e genéticos de seu rebanho, no qual afirmou haver alto grau de endogamia.

Com o objetivo de verificar a presença do SNP c.421G>T no gene ADAMTS2, responsável pela dermatosparaxia em ovinos, foram coletadas amostras de sangue do cordeiro, assim como da sua progenitora e do seu provável progenitor. Em seguida, o DNA genômico foi extraído das amostras sanguíneas com o illustra blood genomicPrep Mini Spin $\mathrm{Kit}^{3}$, seguindo-se as recomendações do fabricante. A genotipagem dos ovinos foi realizada pelo sequenciamento direto de produtos da PCR que amplificava a região do gene ADAMTS2 contendo o SNP c.421G $>$ T responsável pela dermatosparaxia em ovinos da Raça White Dorper. 


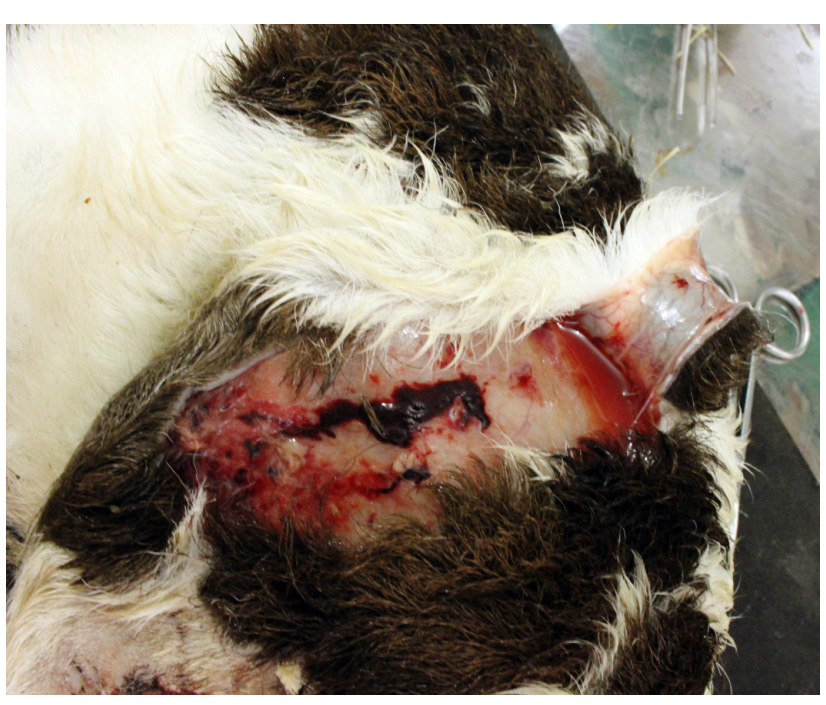

Figura 2. Tecido subcutâneo com edema, focos de hiperemia e hemorragia.

O diagnóstico de dermatosparaxia foi confirmado através da presença do SNP c.421G $>$ T no gene ADAMTS2, em homozigose, no ovino clinicamente afetado. Sua progenitora mostrou-se heterozigota e fenotipicamente normal após o teste genético. O macho testado como possível progenitor era homozigoto dominante (wild type), ou seja, não apresentava alelos mutados associados à doença. Devido ao prognóstico reservado da doença, assim como, à sua característica de herdabilidade, os proprietários optaram pela eutanásia. Após o procedimento, o ovino foi encaminhado para o Setor de Anatomia Patológica Veterinária da UFRRJ.

A necropsia revelou grandes áreas ulceradas em diversas localizações anatômicas, dentre elas, região cervical, esternal, escapular, torácica e face interna e externa das coxas. Notou-se extrema facilidade ao se rebater a pele durante a técnica de necropsia, podendo-se retirá-la até mesmo sem o auxílio de uma faca (Figura 2). No tecido subcutâneo correspondente às áreas ulceradas havia extensas áreas de hemorragia e necrose nas bordas das feridas, que na face posterior dos membros pélvicos deixava exposta a musculatura. Os músculos que compõem a parede abdominal eram muito delgados. As articulações úmero-rádio-ulnar de ambos os membros torácicos estavam aumentadas de tamanho e ao corte deixava fluir líquido amarelo escuro em quantidade moderada. No esterno havia uma extensa área ulcerada típica de escara de decúbito, recoberta por fibrina em organização. À abertura da cavidade torácica, os pulmões apresentavam áreas de hemorragia e infartos multifocais, predominantemente no lobo caudal direito e pequenos focos de atelectasia nas porções cranial e caudal dos lobos esquerdos. Fluía líquido espumoso e vermelho na bifurcação da traqueia em quantidade moderada, indicativos de congestão e edema. À abertura da cavidade abdominal, filamentos de fibrina sobre as vísceras foram observados, além de moderada quantidade de líquido turvo. O lobo esquerdo do fígado estava difusamente pálido, assim como os rins. Todos os segmentos do intestino delgado apresentaram-se moderadamente hemorrágicos e no intestino grosso havia muco nas fezes. Os linfonodos mesentéricos encontravam-se aumentados e úmidos na superfície de corte.

Fragmentos de pele foram coletados de seis regiões anatômicas: cabeça, região cervical, membro torácico, regiões dorsal e lombar e membro pélvico. Posteriormente esses fragmentos foram fixados em formol a $10 \%$ tamponado, processados pela técnica histológica de rotina e corados pela Hematoxilina-Eosina e pelas colorações tricrômicas de Masson e Gomori, e pela coloração por Picrosirius red.

Histologicamente, havia nítida perda da configuração estrutural do colágeno e as fibras de colágeno maduras foram afetadas desde a derme superficial até a profunda. Além disso, eram curtas, de aspecto vítreo-hialinas, e por vezes, fragmentadas ou agrupadas de forma desordenada. As glândulas apócrinas e os vasos linfáticos apresentavam leve a moderada ectasia. Em algumas regiões, como no membro pélvico, havia necrose fibrino-hemorrágica da derme profunda que se estendia à musculatura, e apresentava focos de infiltrado inflamatório composto por macrófagos e plasmócitos, associados a fibroblastos reativos. As colorações tricrômicas de Masson e Gomori foram importantes na evidenciação da displasia das fibras de colágeno (Figuras 3 e 4). A coloração histoquímica com Picrosirius red ressaltou as fibras de colágeno com aspecto fragmentado, de diferentes tamanhos e agrupadas de forma desordenada ao ser incidida pela luz polarizada (Figura 5).

\section{DISCUSSÃO}

O diagnóstico de dermatosparaxia no ovino deste estudo baseou-se nos exames clínicos, anatomopatológicos e moleculares. O diagnóstico molecular foi confirmado através da identificação, em homozigose, do SNP c.421G>T no gene ADAMTS2. Sua progenitora mostrou-se heterozigota e fenotipicamente normal após o teste genético. O macho testado como possível 


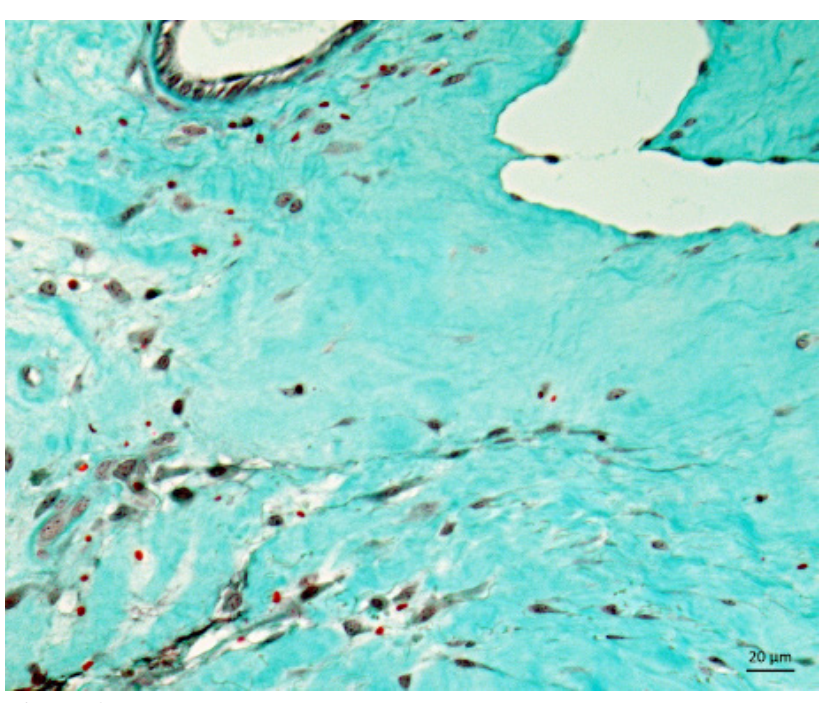

Figura 3. Pele da região lombar esquerda com evidenciação da displasia das fibras de colágeno, Tricrômico de Gomori [obj.20x].

progenitor não era o pai do cordeiro afetado, pois era homozigoto dominante. Segundo o histórico relatado pelo proprietário, um segundo macho (possível progenitor) descartado da propriedade há pouco tempo, filho da progenitora, portanto irmão do cordeiro doente, também poderia ser o pai, porém essa hipótese não pôde ser confirmada, já que o proprietário havia vendido esse animal.

A dermatosparaxia é bem documentada na raça de ovinos White Dorper [2,17,18,20], inclusive no Brasil. Em um estudo realizado por Andrade et al. [3] em 2016, com amostras de sangue de 303 ovinos da raça White Dorper, obtidas de 12 fazendas em diferentes regiões do estado de São Paulo, observou-se que $47(15,5 \%)$ animais tinham um alelo mutado (heterozigotos). No atual estudo, a progenitora era da Raça Santa Inês e os machos da propriedade eram da raça White Dorper.

A dermatosparaxia em animais, em sua forma severa, é caracterizada pela fragilidade cutânea notada após o nascimento [20], o que se assemelha com os achados clínicos observados no presente caso, porém, formas leves da doença também foram relatadas em ovinos da raça Merino, de seis a sete anos de idade, e se caracterizaram pela facilidade de laceração da pele ao manuseio durante o processo de tosquia [6].

Em humanos, além dos achados de hiperextensibilidade e fragilidade cutânea, outros sinais clínicos foram relatados, como frouxidão articular, alterações ósseas, anormalidades vasculares, ruptura uterina e doenças oculares $[12,15]$. Em animais, além da fragilidade cutânea e hiperextensibilidade, essas outras

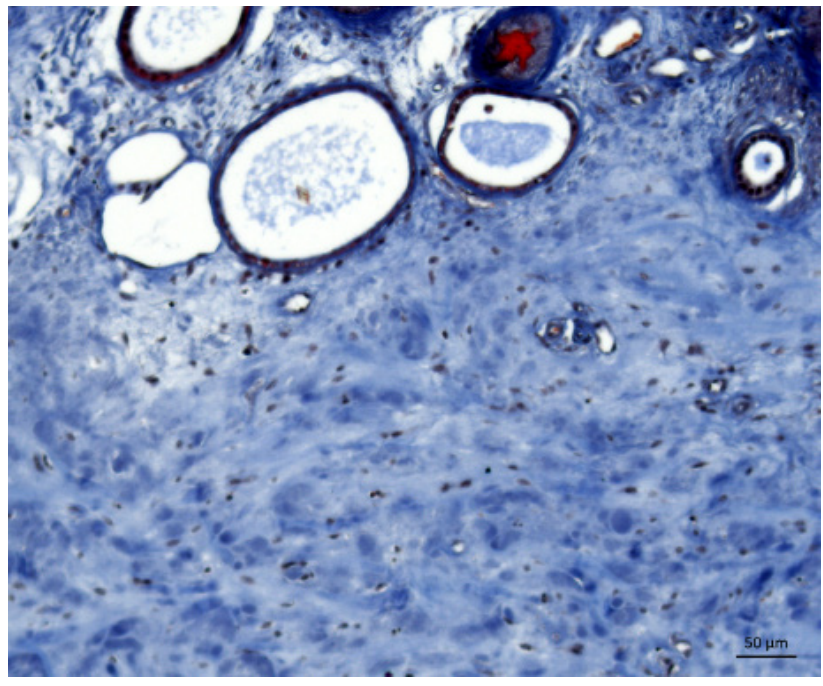

Figura 4. Pele da região dorsal com fibras de colágeno de aspecto homogêneo, Tricrômico de Masson [obj.40x].

alterações clínicas são raramente relatadas [5,9,12]. Embora seja considerada rara, a frouxidão nas articulações úmero-rádio-ulnar dos membros torácicos no ovino do nosso relato, estavam presentes.

Hemorragias foram citadas por Carty [9] no abomaso de dois bezerros; no ovino do nosso estudo também foram observadas extensas áreas de hemorragia em todos os segmentos do intestino delgado e no tecido subcutâneo correspondentes às áreas ulceradas.

Os achados histopatológicos das lesões cutâneas evidenciadas no ovino do presente estudo são compatíveis com as descrições observadas na literatura, quanto às anormalidades da formação do colágeno e desorganização das fibras $[9,18]$. As colorações tricrômicas de Gomori e Masson foram importantes na evidenciação da displasia das fibras de colágeno [9]. Ainda foi feita

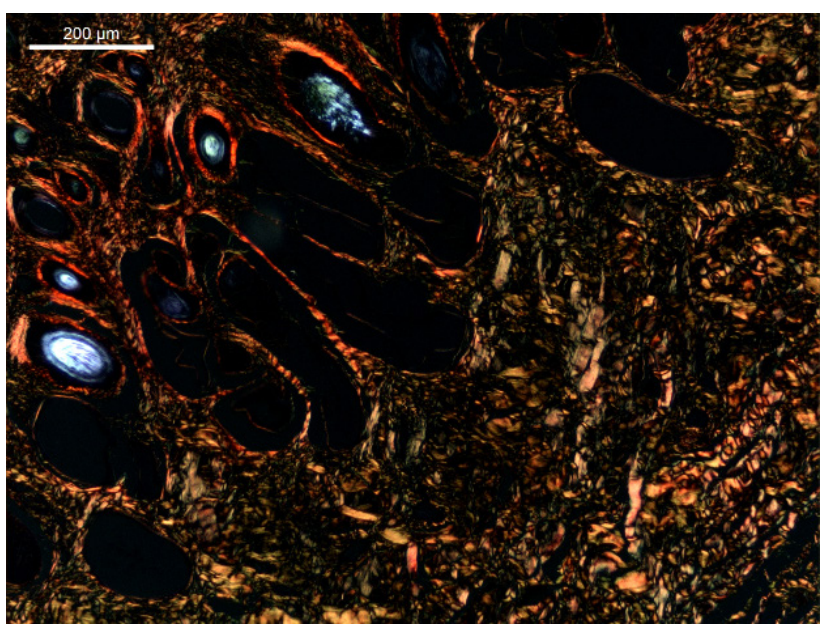

Figura 5. Pele da região dorsal com fibras de colágeno de aspecto fragmentado, de diferentes tamanhos, encurtadas e agrupadas de forma desordenada, Picrosirius red sob luz polarizada [obj.40x]. 
a coloração histoquímica por Picrosirius red, na qual a luz polarizada ressaltou as fibras de colágeno de aspecto fragmentado, menores e agrupadas de forma desordenada e contorcidas, o que se assemelhou em parte com o estudo realizado por Bavinton et al. [6] em 1985.

Baseado no histórico, principalmente relacionado aos acasalamentos pouco controlados no rebanho, assim como, nos achados clínico-patológicos e moleculares foi possível confirmar o diagnóstico de dermatosparaxia no ovino do presente relato. Destaca-se o risco da endogamia nos rebanhos produzir indivíduos heterozigotos, fenotipicamente normais, mas com potencial de gerar cordeiros clinicamente afetados, como o que aconteceu com o ovino do presente relato.

\author{
MANUFACTURERS \\ 'Vallée S.A. Montes Claros, MG, Brazil. \\ ${ }^{2}$ Ourofino Saúde Animal. Cravinhos, SP, Brazil. \\ ${ }^{3}$ Venco Saúde animal. Londrina, PR, Brazil. \\ ${ }^{4} \mathrm{GE}$ Healthcare Life Sciences. Little Chalfont, UK.
}

Declaration of interest. Declaration of interest. The authors report no conflicts of interest. The authors alone are responsible for the content and writing of the paper.

\section{REFERENCES}

1 Andrade S.F., Tostes R.A., Sanches O., Melchert, A., Nogueira R.M.B. \& Valente S.F. 2008. Astenia cutânea em gato (Relato de caso). Ciência Animal Brasileira. 9(2): 524-528.

2 Andrade D.G.A., Pavan L.F., Amorim R.M., Chiacchio S.B., Laufer-Amorim R., Gonçalves R.C., Borges A.S. \& Oliveira-Filho J.P. 2014. Aspectos clínicos, histopatológicos e moleculares da dermatosparaxia em ovinos White Dorper. Pesquisa Veterinária Brasileira. 34(5): 442-448.

3 Andrade D.G.A., Dalanezi F.M., Trecenti A.S., Cunha P.H.J., Borges A.S. \& Oliveira-Filho J.P. 2016. Prevalence study of SNP c.421G>T in the ADAMTS2 gene responsible for dermatosparaxis in White Dorper sheep in Brazil. Pesquisa Veterinária Brasileira. 36(2): 73-76.

4 Badial P.R., Rashmir-Raven A.M., Cagnini D.Q., Oliveira-Filho J.P., Cooley A.J., Cunha P.H.J., Kitchell B.E., Conceição L.G., Mochal C.A. \& Borges A.S. 2013. Marjolin's ulcer in two horses with hereditary equine regional dermal asthenia. Journal Equine Veterinary Science. 33: 515-522.

5 Barnett K.C. \& Cottrell B.D. 1987. Ehlers-Danlos syndrome in a dog: ocular, cutaneous and particular abnormalities. Journal Small Animal Practical. 28: 941-946.

6 Bavinton J.H., Peters D.E. \& Ramshaw J.A.M. 1985. A morphologic study of a mild form of ovine dermatosparaxia. The Journal of Investigative Dermatology. 84: 391-395.

7 Benitah N., Matousek J.L., Barnes R.F., Lichtensteiger C.A. \& Campbell K.L. 2004. Diaphragmatic and perineal hernias associated with cutaneous asthenia in a cat. Journal of the American Veterinary Medical Association. 224(5): 706-709.

8 Borges A.S., Conceição L.G, Alves A.L.G., Fabris V.E. \& Pessoa M.A. 2005. Hereditary equine regional dermal asthenia in three related Quarter horses in Brazil. Veterinary Dermatology. 16: 125-130.

9 Carty C.I., Lee A.M., Wienandt N.A.E., Stevens E.L., Alves D.A., Brownw J.A., Bryan J., Ryan E.G. \& Cassidy J.P. 2016. Dermatosparaxis in two Limousin calves. Irish Veterinary Journal. 69: 15.

10 Damé M.C.F., Riet-Correa F. \& Schild A.L. 2013. Doenças hereditárias e defeitos congênitos diagnosticados em búfalos (Bubalus bubalis) no Brasil. Pesquisa Veterinária Brasileira. 33(7): 831-839.

11 Lapière C.M., Lenaers A. \& Kohn L.D. 1971. Procollagen peptidase: an enzyme excising the coordination peptides of procollagen. Proceedings of the National Academy of Sciences of the United States of America. 68(12): 3054-3058.

12 Mauldin E.A. \& Peters-Kennedy J. 2016. Sistema tegumentar. In: Jubb Kennedy and Palmer's. Pathology of Domestic Animals. 6th edn. v.1. Ontario: Elsevier, pp.543-545.

13 Monteagudo L.V., Ferrer L.M., Catalan-Insa E., Savva D., McGuffin L.J. \& Tejedor M.T. 2015. In silico identification and three-dimensional modelling of the missense mutation in ADAMTS2 in a sheep flock with dermatosparaxis. Veterinary Dermatology. 26:49-e16.

14 Paciello O., Lamagna F., Lamagna B. \& Papparella S. 2003. Ehlers-Danlos - like syndrome in 2 dogs: clinical, histologic and ultrastructural findings. Veterinary Clinical Pathology. 32(1): 13-18.

15 Paepe A. \& Malfait F. 2012. The Ehlers-Danlos syndrome, a disorder with many faces. Clinical Genetic. 82: 1-11.

16Seo S.H., Choi M. \& Hyun C. 2016. Cutaneous asthenia (Ehlers-Danlos syndrome) in a Korean short-haired cat. Korean Journal Veterinary Research. 56(1): 53-55. 
17 Silva A.P.C., Mol J.P.S., Carvalho Junior C.A., Paixão T.A. \& Santos R.L. 2015. Dermatosparaxis in two White Doper lambs in Brazil: case report. Arquivo Brasileiro de Medicina Veterinária e Zootecnia. 67(3): 741-746.

18 Vaatstra B.L., Halliday W.D. \& Waropastrakul S. 2011. Dermatosparaxis in two White Dorper lambs. New Zealand Veterinary Journal. 59(5): 258-260.

19 Van Halderen A. \& Green J.R. 1988. Dermatosparaxis in White Dorper sheep. Journal of the South African Veterinary Association. 59(1): 45.

20 Zhou H., Hickford J.G.H. \& Fang Q. 2012. A premature stop codon in the ADAMTS2 gene is likely to be responsible for dermatosparaxis in Dorper sheep. Animal Genetics. 43(4): 471-473.

21 White S.D., Affolter V.K., Bannasch D.L., Schultheiss P.C., Hamar D.W., Chapman P.L., Naydan D., Spier S.J., Rosychuk R.A.W., Rees C., Veneklasen G.O., Martin A., Bevier D., Jackson H.A., Bettenay S., Matousek J., Campbell K.L. \& Jhrke P.J. 2004. Hereditary equine regional dermal asthenia ("Hyperelastosis Cutis") in 50 horses: clinical, histological, ultrastructural and immunohistological findings. VeterinaryDermatology. 15(4): 207-217. 